\title{
La résilience urbaine en question : Enjeux, contexte et propositions d'opérationnalisation
}

\author{
Urban resilience: Issues, context and operationalization pathways
}

\author{
Charlotte Heinzlef ${ }^{1}$ \\ ${ }^{1}$ Univ.Polynésie Francaise, Ifremer, ILM, IRD, EIO UMR 241, Tahiti, Polynésie francaise, F-98702 FAAA, \\ Charlotte.heinzlef@upf.pf
}

\begin{abstract}
RÉSUMÉ. Face à l'augmentation des risques, leur gestion des risques a évolué progressivement en intégrant de nouvelles approches, telles que le concept de résilience. Concept novateur et pertinent dans l'approche systémique des risques, la résilience peine pourtant à s'opérationnaliser. Ce terme devenu abstrait est peu approprié par les acteurs locaux et reste bien souvent au simple niveau des discours politiques. L'enjeu des recherches actuelles dans la gestion des risques est donc de travailler sur l'opérationnalisation du concept en proposant des outils d'aide à la décision. Cet article présente différentes méthodologies visant à pallier à l'abstraction du concept. Depuis des indicateurs jusqu'à des outils de modélisation ou encore à l'aide d'approches collaboratives, ces approches visent à décortiquer et opérationnaliser la résilience. Pourtant, malgré l'apport de ces méthodologies innovantes, la trop grande diversité des approches ajoute une complexité. Cet article conclue donc sur l'intérêt d'une boîte à outil de résilience sous la forme d'un observatoire.

ABSTRACT. Faced with increasing risks, risk management has gradually evolved by integrating new approaches, such as the concept of resilience. An innovative and relevant concept in the systemic approach to risks, resilience is nevertheless struggling to become operational. This term, which has become abstract, is not very appropriate for local actors and often remains at the level of political discourse. The challenge of current research in risk management is therefore to work on the operationalization of the concept by proposing decision support tools. This article presents different methodologies to overcome the abstraction of the concept. From indicators to modeling tools or collaborative approaches, these approaches aim to operationalize resilience concept. However, despite the contribution of these innovative methodologies, the excessive diversity of approaches adds complexity. This article therefore concludes on the interest of a resilience toolbox in the form of an observatory.

MOTS-CLÉS. résilience, enjeux urbains, évaluation de la résilience, indicateurs, géovisualisation, approches collaboratives, outils d'aide à la décision, boîte à outils de resilience.

KEYWORDS. resilience, urban issues, assessing resilience, indicators, geovisualization, collaborative approaches, decision support tools, resilience toolbox.
\end{abstract}

\section{Introduction}

Dans un contexte de dérèglement climatique, d'augmentation des risques, d'augmentation des incertitudes (Gersonius, 2012; Merad et al., 2016; Zevenbergen et al., 2010), les gestionnaires urbains sont obligés d'innover pour concevoir des stratégies de gestion des risques adéquates. Parmi ces stratégies, rendre les villes résilientes est devenu un impératif. Le concept de résilience est un concept pluridisciplinaire qui définit la capacité d'un système à absorber une perturbation et à récupérer ses fonctions par la suite (Heinzlef, 2019). Cette notion renvoie à une innovation technique, urbaine, sociale, architecturale, économique et politique et enjoint à une remise en question des stratégies traditionnelles de gestion du risque. Cette injonction à l'innovation s'adapte à la complexité spatiale, économique, politique, sociale, écologique du monde contemporain. Pourtant, malgré cette adéquation théorique et conceptuelle, la résilience demeure complexe à intégrer dans les pratiques des urbanistes et acteurs territoriaux. Sa multitude de définitions et d'approches a favorisé son abstraction et son manque d'opérationnalisation.

C'est pourquoi de nombreuses recherches se sont tournées vers l'enjeu d'opérationnalisation, de clarification et d'adoption du concept. Pourtant, le constat est que les outils existants permettant d'améliorer et opérationnaliser la résilience urbaine sont essentiellement tournés autour d'une 
approche technico-fonctionnelle (Lhomme et al., 2013; Serre and Heinzlef, 2018). Or, un territoire est composé de multiples éléments, techniques, urbains et sociaux (Heinzlef et al., 2019). Opérationnaliser la résilience vise à adapter le concept aux diverses composantes qui font partie du territoire. Cet article a pour objectif de présenter les différentes méthodologies qui tentent de répondre aux limites de cette opérationnalisation en favorisant une intégration exhaustive du concept de résilience dans les stratégies de gestion des risques.

Après avoir présenté les enjeux de l'augmentation des risques, nous rappellerons les objectifs et attentes qui se cachent derrière le concept de résilience. Enfin nous développerons différentes approches qui permettent de développer l'opérationnalisation du concept.

\section{Risques et enjeux urbains}

Le contexte de changement climatique a entraîné une augmentation des catastrophes naturelles d'environ 2\% par an depuis une quinzaine d'année (Observatoire permanent des catastrophes naturelles et des risques naturels, 2016). Parallèlement, l'augmentation des personnes et biens en milieux urbains fragilisent considérablement les villes. Or, les zones urbaines produisent entre 70 et $80 \%$ de l'économie mondiale et abritent $50 \%$ de la population mondiale (Zevenbergen et al., 2010). Une telle concentration d'enjeux accroît l'impact des catastrophes et questionne sur l'avenir des villes. Or, cette concentration risque de s'accélérer. Les projections montrent que l'urbanisation, combinée à la croissance globale de la population mondiale, pourrait ajouter 2,5 milliards d'habitants supplémentaires aux zones urbaines d'ici 2050 (United Nations, 2018). La rapidité de ce phénomène, les zones urbaines étant passées de 10\% dans les années 90 à 50\% en seulement deux décennies (Meerow et al., 2016), fragilise le territoire car les villes ne sont pas préparées ou équipées pour faire face aux besoins d'une telle concentration de population. Celle-ci, par manque d'espace disponible, vient s'installer dans des zones à risque (Barroca et al., 2006). Ainsi, une catastrophe sur trois concerne des villes de plus de 500000 habitants (Dauphiné and Provitolo, 2013) Cette croissance urbaine est mal maîtrisée et contenue par les aménageurs et les politiques locaux. Avec des villes qui n'occupent que 1\% du territoire mondial (Dauphiné et Provitolo, 2013), les pays développés n'ont jamais concentré plus de valeur au kilomètre carré (Albouy, 2002) qu'aujourd'hui. Cette concentration de population sur une si petite portion de territoire a accru la vulnérabilité spatiale et sociale par l'exposition des enjeux (Adger and Brooks, 2003). En effet, il semble logique de considérer que plus une population et ses enjeux sont concentrés sur une petite zone, plus les dégâts seront importants. Ce ne sont donc plus seulement la catastrophe en elle-même qui a un impact sur les villes, mais l'urbanisation qui conduit à une sur-vulnérabilité, une vulnérabilité anthropiques, conduisant à une démultiplication de possibilités d'amplification des risques (Mitchell, 1999). Le risque urbain est donc composé de vulnérabilité et d'exposition (Cardona et al., 2012), et la concentration urbaine est donc considérée comme un facteur aggravant dans la gestion du risque. Si nous prenons l'exemple des attentats de 2001, seulement $1 \%$ des bâtiments à New York ont été détruits (Dauphiné et Provitolo, 2013) alors que les pertes humaines ont été très lourdes en raison de la forte densité. Cette densité complique également les services d'urgence, qui peuvent être entravés par la sur-occupation du territoire. Dans ces espaces urbains, on observe l'augmentation des risques naturels mais aussi la création de nouvelles formes de risques (Reghezza-Zitt, 2017). L'architecture interconnectée des activités économiques, sociales, politiques, commerciales, administratives, etc. dans les villes modernes et dans un monde globalisé tend à créer une interdépendance de réseaux (Helbing, 2013), qu'ils soient formels (ex : réseaux techniques) ou informels (ex: interrelations sociales). L'exemple le plus fréquemment cité est la catastrophe de 2005 à la Nouvelle-Orléans (Pescaroli and Kelman, 2017), suite au passage de l'ouragan Katrina en août 2005. L'ouragan et l'onde de tempête ont fait 1833 morts. En raison de l'interdépendance des réseaux, des événements secondaires se sont produits tels que la destruction de réseaux vitaux, l'augmentation et la diffusion de la pollution et la perte de zones stratégiques. Le retour à la normale a été extrêmement compliqué, notamment parce que de nombreux services avaient été détruits, tels 
que les services d'électricité ( 3 millions de citoyens privés d'électricité pendant plusieurs semaines), les réseaux de transport (réduisant la capacité de livraison de nourriture), les réseaux de communication ( 3 millions de clients sans téléphone), mais aussi les infrastructures critiques telles que les services d'urgence et les hôpitaux, laissant des centaines de personnes sans approvisionnement de base (Mancebo, 2006). Dans ce cas, ce n'est pas seulement un risque naturel qui s'est produit, mais une catastrophe qui a eu un impact sur une réalité et une vulnérabilité anthropiques, en raison d'un monde urbain et d'activités interdépendantes. L'interdépendance joue alors le rôle d'un facteur de diffusion du risque. Selon le concept de l'effet de cascade (Nones and Pescaroli, 2016; Pescaroli and Alexander, 2015; Serre and Heinzlef, 2018), c'est-à-dire une réaction en chaîne provoquant des impacts sur un territoire, certaines zones sont touchées par l'aléa, même si elles ne sont pas situées directement dans la zone à risque. Les réseaux agissent alors comme des vecteurs de propagation du risque (Lhomme et al., 2013). Les sociétés et les territoires interconnectés sont donc profondément vulnérables aux éventuelles perturbations fonctionnelles dues à une crise (Boin and McConnell, 2007). Ainsi, si les risques naturels persistent, ils sont transformés par une hybridation sociale, par les actions et les pratiques de l'homme sur son environnement. L'aléa est ensuite modifié par les pratiques urbaines, l'urbanisation et les interactions humaines. Face à cette urbanisation rapide et à l'émergence de ces nouveaux risques, les gestionnaires doivent gérer une série d'incertitudes. Ces incertitudes, liées au changement climatique (Adger et Brooks, 2003), à l'urbanisation (Gonzva et al., 2017, 2015), à la complexité des systèmes (Lhomme et al., 2013), à l'émergence de nouveaux acteurs et de nouveaux risques, compliquent la gestion des risques urbains. Face à ces incertitudes accrues (Gersonius et al., 2013), les réponses apportées par les scientifiques et les gestionnaires sont également en transition. Autrefois orientée vers la gestion des risques (Dobson et al., 2016), elle tend aujourd'hui à intégrer de nouveau concept tel que celui de résilience, en prenant davantage en compte l'aspect systémique d'un territoire.

\section{La résilience, un concept « miracle»?}

Face à ces incertitudes croissantes concernant l'augmentation des risques et leurs impacts en milieux urbains, les experts et gestionnaires urbains doivent innover quant à leurs stratégies de gestion des risques. Le concept de résilience a été intégré progressivement à partir des années 2000 dans ces stratégies.

Depuis une vingtaine d'années, la résilience s'est fait une place dans la gestion des risques (Dauphiné and Provitolo, 2007). Cependant, son usage multidisciplinaire en fait un concept polysémique et abstrait (Bahadur et al., 2010). Ce concept est aujourd'hui sur-utilisé, sollicité dans de multiples domaines (Alexander, 2013) et rattaché à de nombreuses notions (Emrich and Tobin, 2018). Du latin resalire (re, en arrière ; salire, sauter), le terme de résilience est utilisé pour la première fois pour illustrer l'idée de "rebondir» afin de désigner le bruit que fait l'écho en " rebondissant ». De là a découlé plusieurs termes : rebondir, se redresser, résister, réagir, etc. Ces termes peuvent à la fois s'associer ou se contredire, ce qui complexifie la compréhension du concept de résilience et des caractéristiques qui y sont liées. C'est pourquoi, au vu des nombreuses définitions et disciplines associées (Fig.2), il est aujourd'hui plus juste de parler des résiliences que de la résilience (Tisseron, 2015). Cette multitude de « résiliences » s'explique par ses origines mais aussi ses usages divers et variés. Chaque acteur peut apposer sa définition au terme de résilience (Meerow et al., 2016). Cette richesse d'interprétation en fait aussi sa faiblesse, ce qui explique que la traduction de ce concept en stratégies d'action est difficile et laborieuse. 


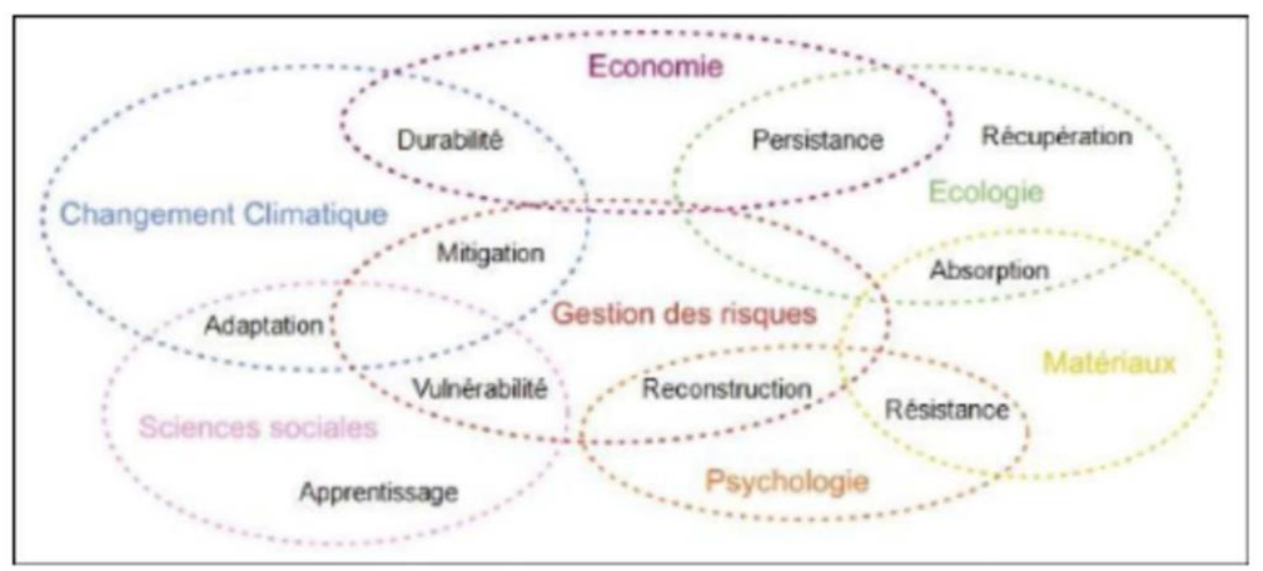

Figure 1. La multitude de disciplines associées au concept de résilience, (Lhomme et al., 2010)

\subsection{La résilience dans la gestion des risques}

La catastrophe de l'Ouragan Katrina à la Nouvelle-Orléans en 2005 a marqué un tournant dans la gestion des risques (Campanella, 2006; Cutter et al., 2008a; Hernandez, 2010). Pour éviter qu'un événement pareil se reproduise, la gestion des risques a évolué en intégrant le concept de résilience. L'objectif est d'utiliser ce concept pour préparer au mieux les populations et territoires à l'augmentation des risques en milieu urbain. L'idée n'est plus d'analyser les risques de manière cloisonnée mais d'étudier l'événement perturbateur et ses conséquences dans sa globalité.

Aujourd'hui, l'acceptation scientifique qui analyse la ville comme un système (Bretagnolle et al., 2006; Gardner, 2016; Ruth and Coelho, 2007), fait écho à l'approche systémique de la résilience. C'est pourquoi la résilience fait aujourd'hui partie de la panoplie de concepts utilisés dans la gestion des risques. Présentée par les acteurs internationaux (UN General Assembly, 2015; United Nations Office for Disaster Risk Reduction (UNDRR), 2017) comme la clef de voute de la gestion des risques, la résilience est aujourd'hui une priorité d'action. La résilience urbaine se définie comme la capacité d'un système urbain à s'adapter aux perturbations. Elle représente donc le nouveau paradigme de la gestion des risques qui permet d'atténuer l'impact négatif d'une perturbation. La résilience urbaine conduit à la création d'une ville plus durable (Balsells, 2015; Serre et al., 2013). Ce modèle met en avant l'idée que la perturbation - ou choc - n'est pas (ou plus) nécessairement quelque chose de négatif mais participe pleinement à la construction d'un nouveau modèle en appuyant l'idée d'innovation, d'apprentissage, de rebond et de changement.

\subsection{Les tensions liées au concept}

Opérationnaliser la résilience est un sujet complexe, voire conflictuel. Du fait de son origine pluridisciplinaire, et de la multitude d'approches, les interprétations de la résilience et son opérationnalisation sont parfois contradictoires (Quenault, 2013). Cette contradiction tient essentiellement du fait que la résilience appartient à de nombreuses disciplines, la physique, la psychologie, l'écologie ou encore la gestion des risques (Alexander, 2013). Ce flou disciplinaire et conceptuel rend complexe l'utilisation de la résilience et son intégration dans des stratégies de gestion des risques (Dauphiné and Provitolo, 2007). Le concept de résilience est confronté à un problème de formalisation ce qui rend difficile son passage de la théorie à la pratique (Djament-Tran et al., 2011). Malgré son succès grandissant, la pertinence opérationnelle du concept est donc en permanence remise en doute et questionnée (Dauphiné and Provitolo, 2004; Djament-Tran et al., 2011). Opérationnaliser la résilience passe nécessairement par sa (re)définition et son évaluation (Dauphiné and Provitolo, 2007). Il est donc nécessaire de produire des indicateurs adéquats afin « d'objectiver la résilience » (Djament-Tran et al., 2011). Or, comme chaque approche sur ce concept est différente, définissant la résilience comme, un processus, un état, une qualité ou un objectif, le terme de résilience est dès lors subjectivé par les utilisateurs. 
Comment donc opérationnaliser, et donc objectiver, le concept tout en gardant sa richesse d'interprétations ? Certaines approches associent également d'autres concepts comme la vulnérabilité (Burton et al., 1993). Selon certaines écoles la résilience est une des composantes de la vulnérabilité (Provitolo and Reghezza-Zitt, 2015) tandis que pour d'autres la vulnérabilité représente l'incapacité à faire face à un choc et est donc la contradiction de la résilience (Becerra and Peltier, 2009). Cette difficile formalisation, liée à la multitude d'interprétations et d'approches, a pour conséquence un passage de la théorie à la pratique complexe. Pourtant, c'est le défi posé par toutes les études portées sur la résilience, afin d'utiliser ce concept pour construire des stratégies de gestion des risques adéquates. Plusieurs approches ont donc tenté de répondre à ces enjeux en proposant des méthodologies qui visent à opérationnaliser la résilience. Cette opérationnalisation se traduit par la conception d'outils de mesure de résilience, de systèmes spatiaux d'aide à la décision ou encore par des approches favorisant la collaboration entre experts et acteurs locaux. Nous analyserons dans cette partie certains de ces travaux, cadres, structures et méthodologies. Certains auteurs ont tenté de synthétiser tous les modèles existants (Constas et al., 2010; Schipper and Langston, 2015) mais la quarantaine de modèles évoquée (Bahadur et al., 2015) souligne la (sur)abondance des approches sur la résilience. Nous nous attacherons quant à nous à balayer les approches visant à l'évaluation de la résilience via la création d'indicateurs, les modèles proposant un cadre conceptuel ou des systèmes d'aide à la décision, puis les méthodologies visant à créer un travail collaboratif afin d'opérationnaliser la résilience.

\section{Enjeux et stratégies d'opérationnalisation du concept de résilience}

L'opérationnalisation consiste en grande partie à déterminer comment un concept peut être mesuré (Adger et al., 2004) et à déterminer quels seront les indicateurs qui serviront à mesurer le concept afin de produire des données à son sujet. L'évaluation de la résilience passe donc essentiellement par sa mesure (Dauphiné and Provitolo, 2007) et la création d'indicateurs.

\subsection{Evaluer la résilience à l'aide d'indicateurs}

Les indicateurs sont des variables quantitatives destinées à représenter une caractéristique d'un système ou concept. Ils ont été employés pour éclairer la prise de décision, améliorer la participation des parties prenantes, établir un consensus, explorer les processus sous-jacents etc. (Parris and Kates, 2003). L'objectif d'un indicateur est d'apporter « une information devant aider un acteur, individuel ou plus généralement collectif, à conduire le cours d'une action vers l'atteinte d'un objectif ou devant lui permettre d'en évaluer le résultat » (Bonnefous, 2001). L'indicateur peut être un paramètre, une valeur, une donnée ou encore une observation. Son objectif est de donner des indications ou décrire un phénomène, une situation, un environnement, un processus.

Plusieurs méthodologies se sont appuyées sur les indicateurs comme outil d'opérationnalisation du concept de résilience (Heinzlef et al., 2019).

- Le Baseline Resilience Indicators for Communities (BRIC) (Cutter et al., 2014)

Susan Cutter a développé un modèle mettant en relation la vulnérabilité et la résilience (the Disaster Resilience of Place, DROP, (Cutter et al., 2008b)), puis une base de données d'indicateurs de résilience, le BRIC (Baseline Resilience Indicators for communities) (Cutter et al., 2014). Le BRIC (Baseline Resilience Indicators for Communities), a cherché à disséquer les notions et caractéristiques liées au concept de résilience. Divisant la résilience en six indicateurs - social, économique, communautaire, institutionnel, infrastructurel et environnemental - Cutter se propose de mesurer la résilience (Cutter et al., 2014). Chaque indicateur est divisé en sous variables telles que l'éducation, l'âge, la maîtrise de la langue, le taux d'emplois, le taux d'immigration, l'accès à la nourriture, l'entraînement aux catastrophes, la stabilité sociale, l'accès à la santé, l'accès aux énergies, etc. Chaque variable a un effet positif ou négatif sur la résilience communautaire. Ces 
indicateurs ont permis de cartographier la résilience à l'échelle des Etats-Unis (Fig.4) et d'analyser les composantes qui pouvaient permettre une augmentation du potentiel de résilience à l'échelle nationale.

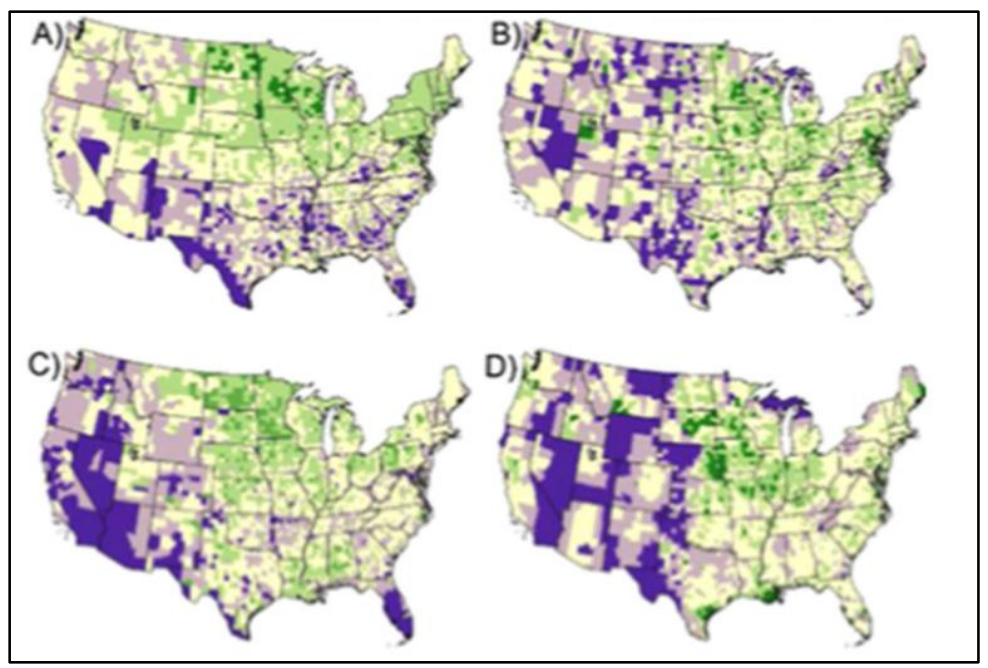

Figure 2. Cartographie de la résilience à l'échelle des Etats-Unis par indicateur: $A=$ social, $B=$ économique, $C=$ communautaire, $D=$ institutionnel (Cutter et al., 2014)

- Le DS3 Model (Spatial Decision Support System) (Serre, 2018)

Le DS3 Model a permis de définir trois capacités pour évaluer la résilience des réseau urbains face à un risque inondation. La résilience est définie ici comme la capacité d'un système à absorber une perturbation et à récupérer ses fonctions par la suite. Trois capacités sont supposées déterminer du degré de résilience de ces réseaux (Fig.4) :

- La capacité de résistance : elle consiste à déterminer les dommages matériels suite à un risque. Il est considéré que plus un réseau est endommagé, plus il y aura une probabilité de dysfonctionnement et plus la remise en service sera lente et complexe ;

- La capacité d'absorption : elle illustre les fragilités et forces du réseau permettant de construire des alternatives à celui-ci à la suite d'une défaillance de composants ;

- La capacité de récupération : elle représente le temps nécessaire à la remise en service du réseau et de ses composants.

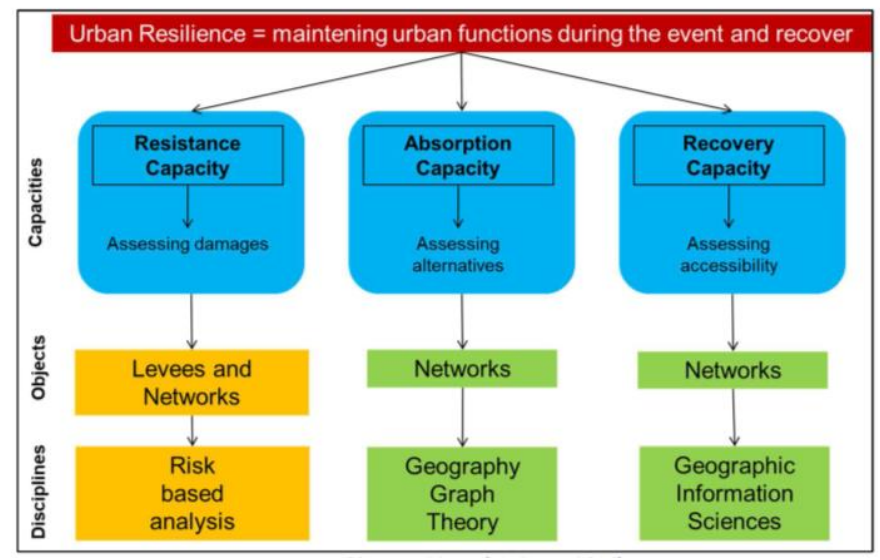

Figure 3. Le DS3 Model (Serre, 2018)

- Global Resilience Indicators (Heinzlef et al., 2019 ; Heinzlef et al., 2020a)

Les Global Resilience Indicators ont permis d'établir une approche exhaustive du concept de résilience à travers trois indicateurs de mesure, un indicateur de résilience sociale, de résilience 
urbaine et de résilience technique (Fig.6). L'objectif a été d'appréhender la complexité systémique du territoire à travers l'analyse de la résilience.

- L'indicateur de résilience sociale: La résilience sociale peut être définie comme la capacité d'une population à s'adapter et à se remettre des perturbations (Hutter et Lorenz, 2018). De nombreux facteurs peuvent permettre à une entité sociale d'agir, de réagir et de redévelopper de manière proactive des activités ou des interactions, tels que l'âge (Cutter et al., 2010), le statut socioéconomique (Flanagan et al., 2011), la connaissance et la perception des risques, la diversité, etc. Dans cette étude, nous entendons par résilience sociale la résilience de la communauté sociale (Wilson, 2013) et non la résilience individuelle (Hutter et Lorenz, 2018).

- L'indicateur de résilience urbaine: Nous considérons que l'indicateur de résilience urbaine comprend toutes les dynamiques urbaines - physiques comme les bâtiments (âge des bâtiments, densité, fonctionnalité) ou les infrastructures critiques - ou virtuelles comme les dynamiques économiques, évaluées par la création ou la disparition de nouvelles entreprises. Nous avons établi quelques variables pour comprendre le degré de résilience urbaine, en tenant compte de la zone d'influence des infrastructures critiques (pompiers, police, forces de défense), de l'accès à la santé (Norris et al., 2008 ; Opach et Rød, 2013), de la densité des logements sociaux (Johansson et al., 2016), de la dynamique touristique (Tierney, 2009) et de la dynamique économique (création de nouvelles entreprises, maintien des anciennes).

- L'indicateur de résilience technique: Nous entendons par variables techniques tous les éléments matériels interconnectés tels que les réseaux urbains. Les réseaux de transport, de gaz, d'électricité, d'eau (leur âge, leur capacité de résistance, d'absorption, de récupération (Serre, 2016) sont intégrés dans cet indicateur. En effet, des recherches récentes mettent en évidence l'impact (négatif ou/et positif) des réseaux urbains pendant et après une crise. Ils sont essentiels au bon fonctionnement d'une ville, reliant de plus en plus de personnes et de territoires et, de ce fait, offrant une variété de ressources et d'opportunités mais ils peuvent aussi créer des situations d'interdépendance (Serre et Heinzlef, 2018). Elles peuvent, par leurs caractéristiques d'interconnexion, propager les inondations au-delà des frontières géographiques et fonctionnelles (Boin et McConnell, 2007). Nous analysons donc ici l'accessibilité et la diversité des réseaux urbains dans un rayon de $100 \mathrm{~m}$.

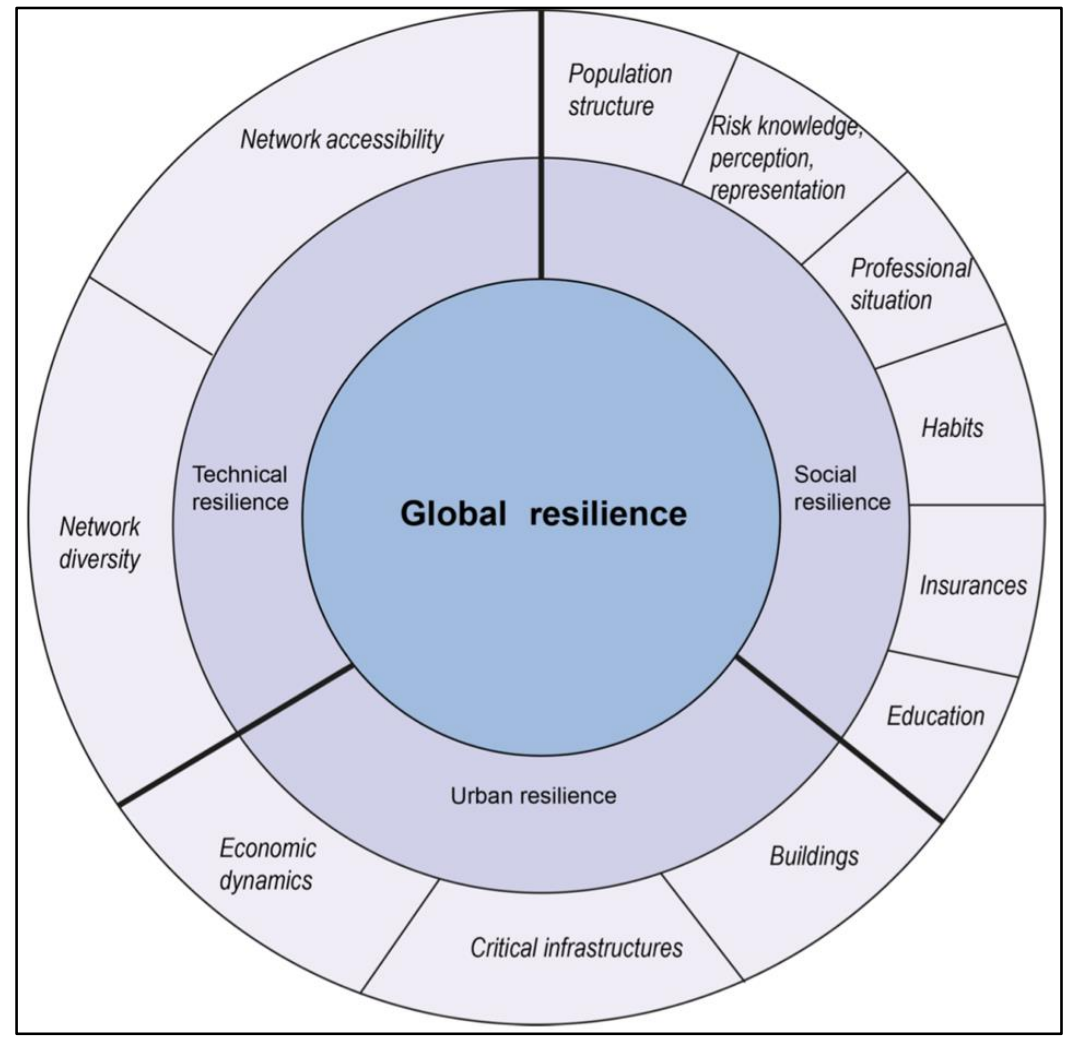

Figure 4. Indicateurs de résilience globale (Serre et Heinzlef, 2018) 
Ces indicateurs permettent de décortiquer le concept de résilience en identifiant des critères, capacités et caractéristiques qui lui sont propres. Que ce soit avec des approches davantage tournées autour de la vulnérabilité (SoVI) ou autour de la résilience technique (DS3 Model) ou globale (BRIC et Global Resilience Indicators), les méthodologies ont toutes pour objectif d'aiguiller sur les éléments à approfondir ou développer afin d'augmenter la résilience territoriale. Ces indicateurs se complètent bien souvent par des outils spatiaux afin de géovisualiser les potentiels de résilience à des échelles plus ou moins fines.

\subsection{Opérationnaliser la résilience à l'aide de technique de géovisualisation}

Le concept de résilience étant un sujet complexe à traiter et à opérationnaliser pour les acteurs locaux, de nombreux outils ont été créés pour simplifier, définir, mesurer et tenter d'opérationnaliser ce concept. La nécessité de créer des systèmes d'aide à la décision prend tout son sens vis à vis de l'abstraction du concept. Lorsque des questions territoriales sont abordées, on parle alors de systèmes spatiaux d'aide à la décision (SSAD). Ces SSAD permettent d'apporter une aide informatisée afin d'élaborer et évaluer des scénarios, stratégies et interventions adéquates face à un problème spatial. Ils permettent d'acquérir, condenser, travailler, analyser et valoriser des données/informations géographiques et données géo-spatiales (Marzouki et al., 2017). L'intégration de la visualisation dans l'analyse de données géo-spatialisées a conduit à une transformation de la cartographie traditionnelle grâce à la géovisualisation (Çöltekin et al., 2017) et à favoriser la compréhension de notions complexes. La géovisualisation combine la visualisation scientifique, la visualisation de l'information, la cartographie, les systèmes d'information géographique (SIG), l'analyse exploratoire des données et bien d'autres méthodes pour explorer, analyser, synthétiser et représenter les données et informations géographiques (Nöllenburg, 2007). C'est pourquoi de nombreux systèmes d'aide à la décision spatiale ont été dotés des techniques de visualisation et d'interfaces dynamiques afin d'associer capacités technologiques et interprétations et savoirs locaux.

Plusieurs méthodologies ont permis de produire des outils afin de clarifier les concepts de résilience et de vulnérabilité. Ces outils sont des systèmes d'aide à la décision spatiale et ont permis de décortiquer le concept de résilience à travers un processus de visualisation. L'objectif de chacune de ces approches est de rendre accessible le concept en créant des liens entre avancées scientifiques et réalité territoriale.

- L'outil DOMINO (Robert et al., 2017 ; Heinzlef et al., 2020b)

Un outil de modélisation de la propagation dans l'espace et dans le temps des effets domino entre les infrastructures critiques (IC) a été développé sur le territoire de la ville de Montréal (Robert and Cloutier, 2012; Robert and Morabito, 2009). Il consiste en une base de données géographique dans laquelle les organisations ont saisi les informations pertinentes relatives à leurs dépendances aux ressources essentielles qu'elles utilisent. Des modules, créés sur la structure des systèmes experts, permettent de combiner les informations provenant des différentes organisations afin d'identifier les interdépendances entre elles. Un simulateur temporel a aussi été développé afin de permettre de visualiser la propagation des effets domino potentiels à la suite d'une défaillance (Figure 7). S'agissant d'un outil géomatique, il est possible de combiner plusieurs couches d'informations dans DOMINO. Les partenaires (gestionnaires des IC et responsables de la sécurité civile) y ont un accès sécurisé et géré selon des niveaux correspondant à leur profil d'utilisateur. Ainsi, chaque organisation a accès à ses propres informations alors que les résultats des simulations sont accessibles à tous les utilisateurs. 


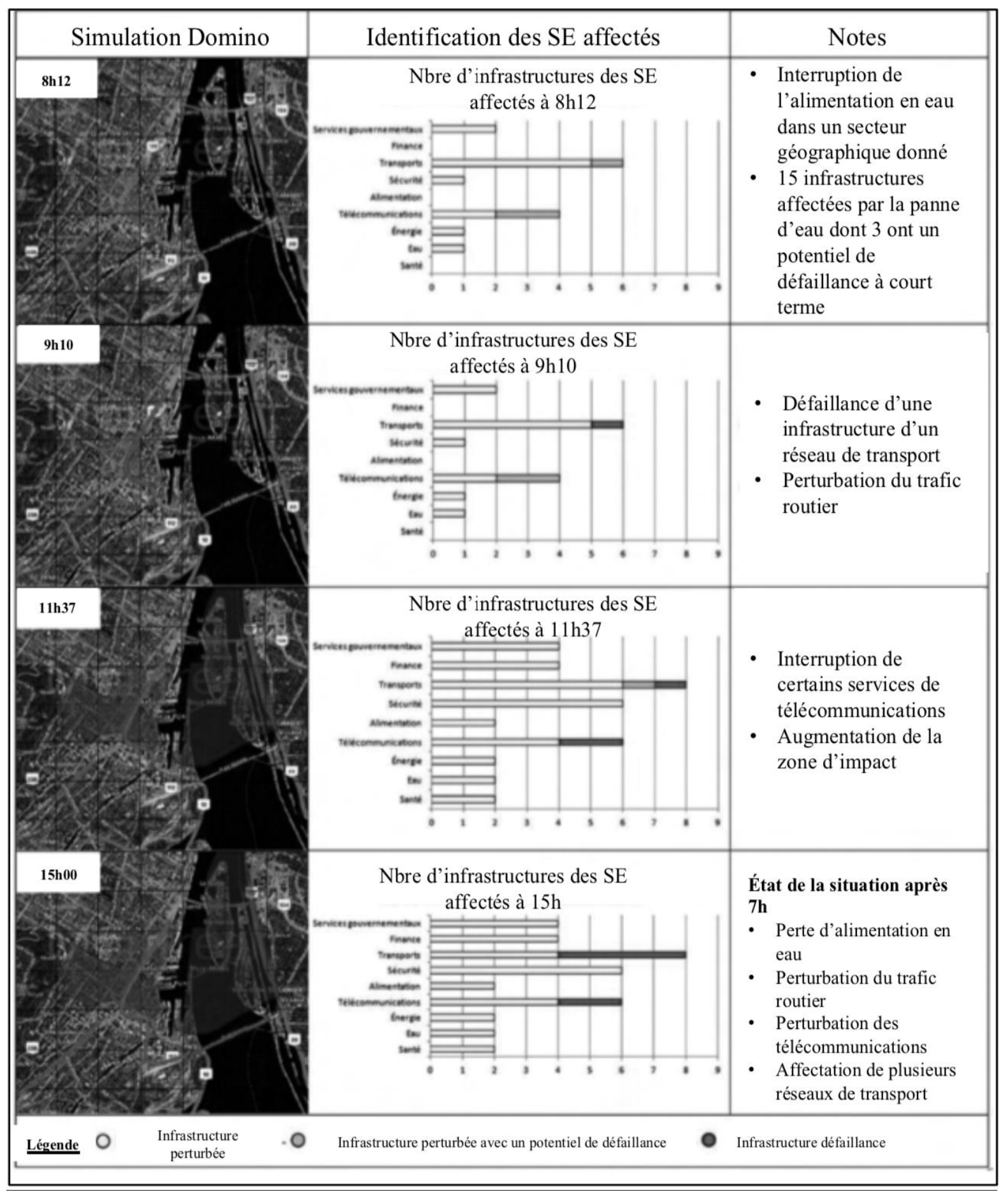

Figure 5. Exemple de l'outil DOMINO et de la propagation du risque d'inondation et des défaillances (Robert et al.,2017)

En termes de résilience, DOMINO permet des analyses sur les différents paramètres de la résilience. Son implantation sur un territoire demande aux diverses organisations concernées d'échanger des informations sur leur propre capacité de gestion des perturbations. La mise en commun de ces informations permet de réaliser des analyses de cohérence sur un territoire et d'intégrer des conséquences plus larges sur la communauté.

- Le XDI Sydney project (NWS government and Australian climate tech company XDI Cross Dependency Initiative, 2018)

Le projet XDI a pour objectif de quantifier l'impact des risques climatiques sur les infrastructures critiques du Grand Sydney (Australie) et d'identifier les interdépendances et les potentiels effets 
domino. L'idée principale est de quantifier les coûts liés aux impacts du changement climatique sur les infrastructures critiques entre 2019 et 2100 (Fig.8). Parallèlement à cette quantification, ce projet a pour volonté de proposer une approche standardisée de la résilience et de son opérationnalisation à travers les différents secteurs d'analyse et d'utilisation. Grâce à une identification des espaces à risques mais également des opportunités, l'enjeu est de développer une approche collaborative et d'adaptation. Enfin, l'amélioration des plans de gestion et d'investissement des infrastructures est visée.

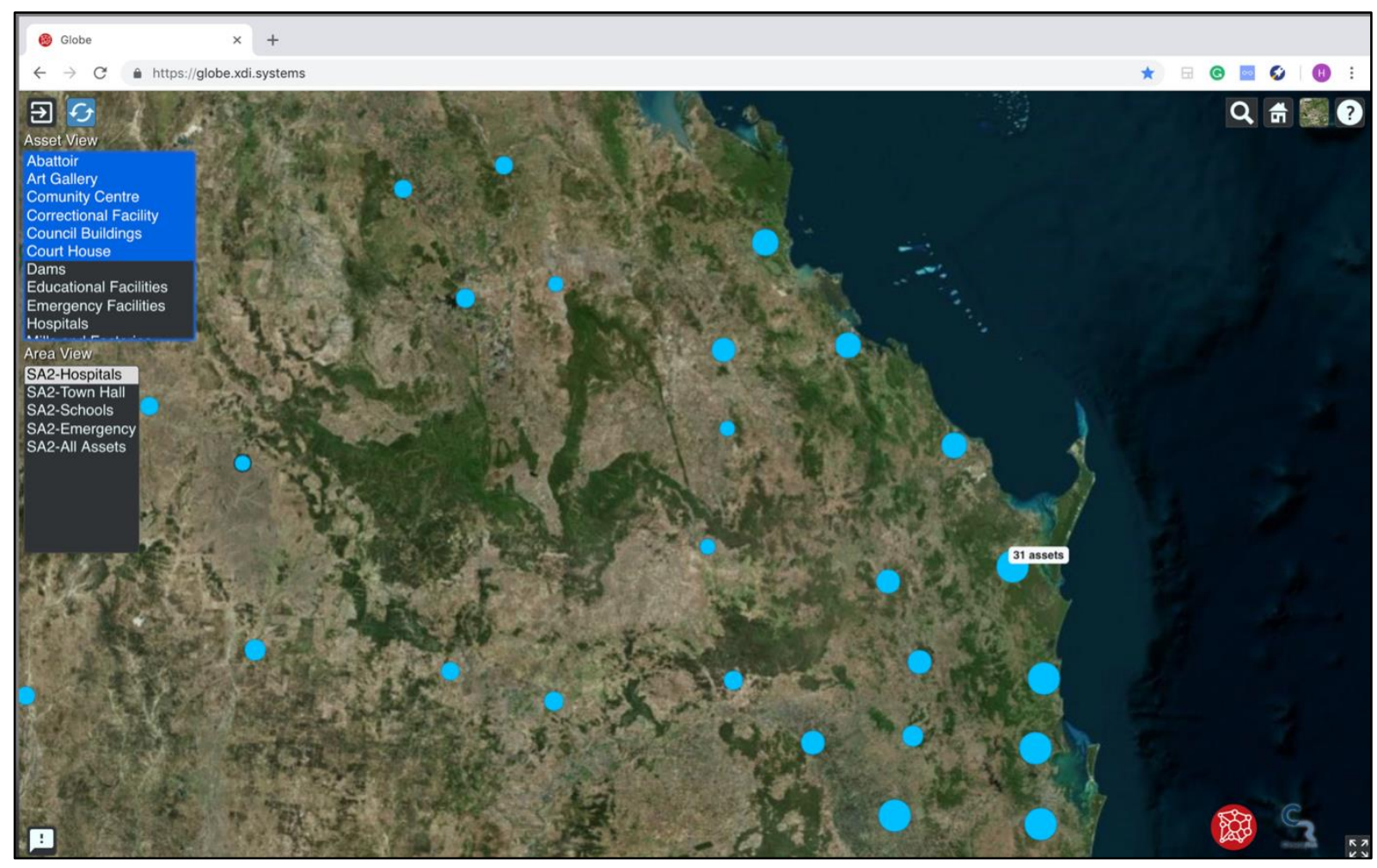

Figure 6. Exemple de la plateforme XDI pour cartographier les infrastructures critiques (NWS government and Australian climate tech company XDI Cross Dependency Initiative, 2018)

La plateforme XDI a plusieurs avantages :

- une puissante plateforme en qui calcule et affiche l'analyse des risques climatiques.

- Elle rassemble des données sur les actifs, des cartes de risques géospatiales, des projections d'impact du changement climatique, des données d'ingénierie et des analyses financières.

- fournit une identification sophistiquée des risques et une analyse coûts-avantages pour la planification de l'adaptation dans tout le spectre des infrastructures.

- soutient la prise de décision lors de l'examen des options d'investissement en matière de résilience en comparant les coûts d'investissement aux coûts d'exploitation économisés et aux dépenses encourues par les pertes ou les assurances.

Les utilisateurs accèdent à la plateforme via une connexion sécurisée et travaillent avec leurs données personnelles dans un environnement interactif qui permet une identification rapide des hotspots de risque. Les utilisateurs peuvent approfondir leurs recherches pour obtenir des informations sur les actifs par risque, élément d'ingénierie ou zone géographique.

- The Coastline Resilience (2007)

L'outil Coastline Resilience est un outil virtuel d'aide à la décision afin d'apporter des réponses aux effets du changement climatiques et des risques. Cet outil a été conçu à l'aide d'une approche 
collaborative entre plusieurs acteurs (The Nature Conservancy, United Nations University, Association of State Floodplain Managers, University of Southern Mississipo, Esri, Global Disaster Preparedness Center, International Federation of the Red Cross, etc.). L'outil "Coastal Resilience" comprend une plateforme de visualisation où les informations écologiques, sociales et économiques peuvent être consultées parallèlement à des scénarios d'élévation du niveau de la mer et d'ondes de tempête dans des zones géographiques spécifiques. En outre, une architecture modulaire et configurable de plugins permet à des régions géographiques spécifiques de disposer d'applications conçues spécialement pour le géo-traitement et l'affichage. Ces applications répondent aux besoins des parties prenantes, des politiques et des processus de planification. Les applications sont utilisées pour simplifier des relations ou des modèles complexes, pour transmettre un concept écologique ou social spécifique, ou pour comparer différents scénarios de conditions futures. Plusieurs variables sont étudiées et cartographiées, telles que la vulnérabilité sociale (population âgée de moins de 5 ans ou de plus de 65 ans, risque d'inondation par ruissellement ou encore risque d'inondation côtière (Fig. 9)).

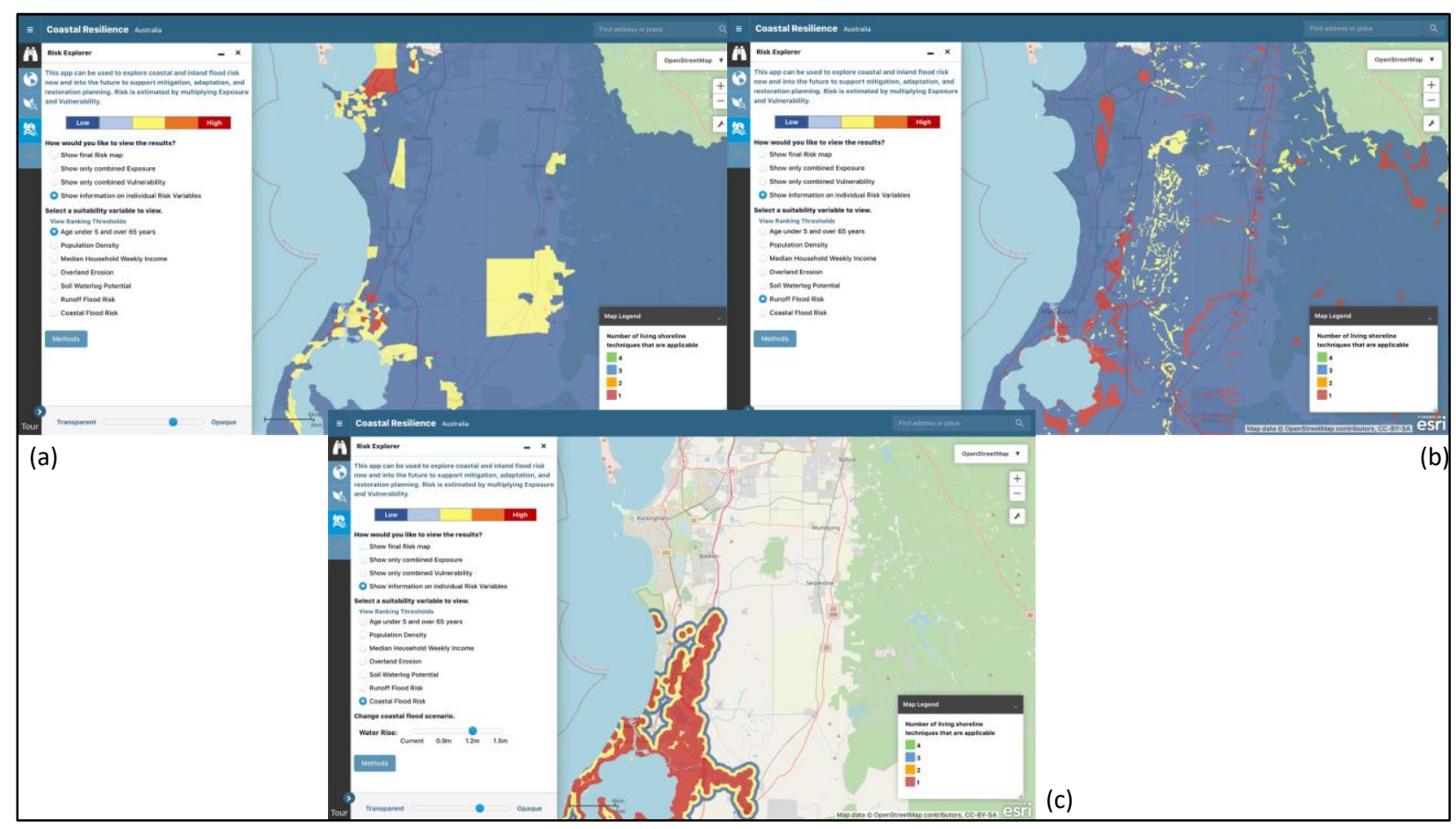

Figure 7. Interface du Coastline Resilience en Australie au sud de Perth. (a) la vulnérabilité sociale avec les populations âgées de moins de 5 ans et plus de 65 ans, (b) le risque inondation par ruissellement et (c) le risque d'inondation côtière (The Coastline Resilience, 2014)

Opérationnaliser la résilience passe par plusieurs approches. La conception de systèmes spatiaux d'aide à la décision fait partie de l'une d'elles. Afin de favoriser la compréhension et l'appropriation de concepts complexes et abstraits, ces systèmes ont été dotés des techniques de visualisation et d'interfaces dynamiques. Cette combinaison de cartographie et de visualisation scientifique, de visualisation de l'information, des systèmes d'information géographique (SIG), de l'analyse exploratoire des données se nomme la géovisualisation. Grâce à une interface dynamique et à une puissance technique capable de traiter des données complexes, les outils de géovisualisation permettent de communiquer des informations nécessaires au processus d'aide à la décision. Les différentes approches présentées ci-dessus adoptent ces techniques. Parallèlement à la conception de ces systèmes spatiaux d'aide à la décision, des ateliers sont souvent organisés, en parallèle ou a posteriori de la conception de l'outil. Ces ateliers amorcent une démarche collaborative afin de favoriser et d'entraîner une prise de décision. Or, malgré une expertise de la part des acteurs dans le processus d'aide à la décision, ce même processus nécessite l'aide des bénéficiaires (Merad et al., 2014). En d'autres termes, le processus d'aide à la décision à lui-même besoin d'aide de la part des 
acteurs locaux. C'est pour développer et soutenir le « processus créatif individuel et/ou collectif » de l'aide à la décision qu'une méthodologie collaborative s'est développée.

\subsection{Développer des approches collaboratives afin de participer à l'opérationnalisation de la résilience}

Le United Nations International Strategy for Disaster Risk Reduction (UNISDR) a développé 10 points essentiels afin de créer des villes résilientes. Le point $n^{\circ} 1$ est de mettre en place des organisations ou coordinations afin de comprendre et réduire les risques, basées sur la participation des citoyens et sociétés civiles (UNISDR, 2012). L'objectif est de construire des actions et alliances locales afin de s'assurer que chaque acteur comprend son rôle dans la réduction et la préparation de stratégies de réduction des risques et de résilience.

Depuis quelques années, la question des risques et plus particulièrement des risques environnementaux revient fréquemment sur la scène politique et publique. Grâce aux termes « justice environnementale », « développement durable », « équité environnementale », ou encore " services écosystémiques », les questions et les intérêts des citoyens se font plus présents. Impliqués de plus en plus dans des questions environnementales mais aussi humaines et citoyennes, les populations sont responsabilisées sur leur mode de vie. Or, ces implications ne peuvent perdurer que si un travail de transparence, de clarté, de communication et de consensus s'établi entre les différents acteurs (Mustajoki et al., 2004). Car du fait de la complexité des enjeux environnementaux, liés eux-mêmes aux enjeux urbains, la communication, la compréhension mais aussi nécessairement l'intégration et l'action ne peuvent exister que par l'accompagnement des experts et acteurs locaux.

C'est donc un changement de paradigme qui doit s'opérer afin d'intégrer les parties prenantes. Si cette transition peut paraître insurmontable pour certains, non productif pour d'autres, il est pourtant avéré que la construction d'un savoir commun aux experts et profanes " permet de réduire la complexité, de prendre en compte les intérêts de chacun, mais aussi de favoriser l'acceptation et l'appropriation des solutions » (Toubin, 2014). Ainsi malgré les difficultés pour instaurer cette confiance, cette collaboration, ce dialogue commun, le résultat est une meilleure intégration et acceptation du travail des experts. En outre, étant donné que ces sujets tels que les questions environnementales, les risques urbains, le changement climatique, touchent et impliquent toutes les parties prenantes, les intégrer permet non seulement d'aborder de nouveaux points de vue et approches mais aussi de construire une équité de connaissances. Ainsi donc, l'intégration des différents acteurs a un impact doublement positif, et permet de répondre aux objectifs d'un projet en améliorant ses propositions, mais est également un objectif en soi en répondant aux désirs de la concertation. Il est alors question pour les gestionnaires de "s'assouplir pour s'adapter » et de " négocier pour convaincre » (Joerin, 1997) afin de formaliser dès le début du projet une vision et un savoir co-construits entre les différents acteurs. De ce fait, le résultat sera d'autant plus légitime et surtout pertinent (Desthieux, 2005). Ainsi, par le dialogue, le compromis, la sensibilisation des individus, les stratégies d'adaptation face aux risques auront encore plus de chances d'avoir un impact positif et réel sur le territoire et sa population.

L'opérationnalisation de la résilience passe donc nécessairement par l'implication des multiples acteurs afin de proposer un urbanisme cohérent et des stratégies d'adaptation pour répondre au mieux à la menace des risques croissants en milieux urbains. Les politiques publiques peuvent dès lors jouer un rôle essentiel à la mise en place d'approches collaboratives. En effet, il est aujourd'hui obligatoire de mettre en place une forme de concertation lors de la conception de chaque nouveau projet urbain (Mermet, 2006). La concertation, «action (...) partiellement négociée, partiellement débattue et délibérée» (Mermet, 2006), se retrouve alors comme condition sine qua non d'un projet du territoire. 
Plusieurs approches ont cherché à développer et mettre en avant les apports d'approches collaboratives dans le processus d'opérationnalisation de la résilience.

- Les 100 villes résilientes (The City Resilient Framework, 2014)

Créé par la fondation Rockfeller en 2013, les 100 Villes Résilientes ont pour objectif d'aider les villes du monde entier à être davantage résilientes face aux enjeux physiques, sociaux et économiques du $21^{\mathrm{eme}}$ siècle. Ce modèle (Fig.10) permet aux villes membres de bénéficier d'accompagnement technologique, financier, technique et d'une expertise scientifique afin d'analyser, planifier et mettre en œuvre des stratégies de résilience urbaine. Le 100RC soutient l'adoption et l'intégration d'une vision de la résilience qui inclut non seulement les catastrophes naturelles - tremblements de terre, incendies, inondations, etc. - mais aussi les tensions qui affaiblissent le tissu urbain d'une manière quotidienne ou cyclique. Ces contraintes ou stress peuvent par exemple être un chômage élevé ; un système de transport public surchargé ou inefficace ; une violence endémique ; ou des pénuries chroniques de nourriture et d'eau. En s'attaquant à la fois aux chocs et aux stress, une ville devient plus apte à réagir aux événements indésirables et, dans l'ensemble, elle est mieux à même de fournir des fonctions de base, à la fois bonnes et mauvaises, à toutes les populations.

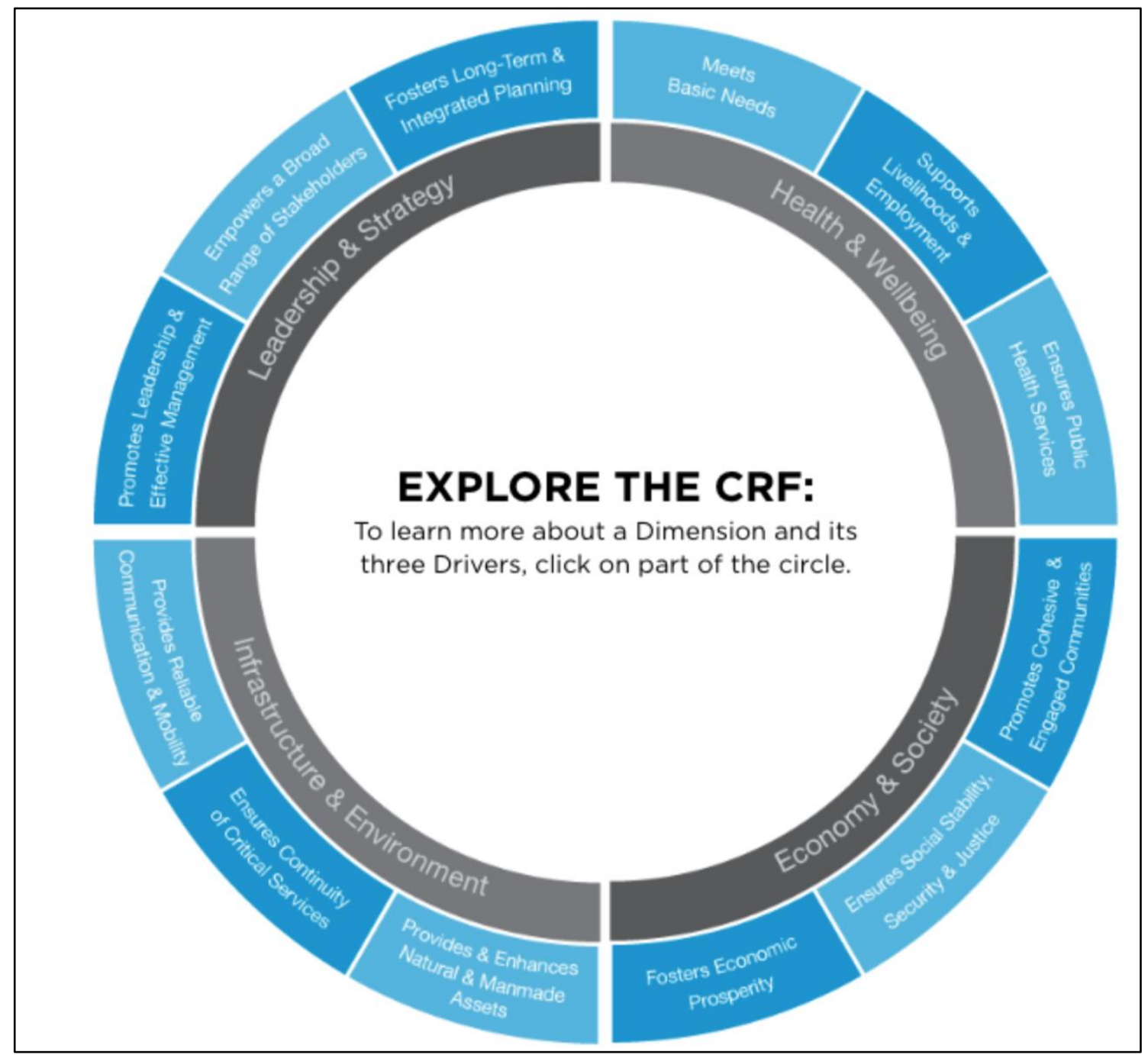

Figure 8. Modèle conceptuel des 100 Villes Résilientes (The City Resilient Framework, 2014)

Les villes du réseau 100RC disposent des ressources nécessaires pour développer une feuille de route vers la résilience selon quatre angles d'approche : 
- Orientation financière et logistique pour l'établissement d'un nouveau poste innovant dans le gouvernement de la ville, un chef de la résilience, qui dirigera les efforts de résilience de la ville

- Soutien d'experts pour le développement d'une solide stratégie de résilience

- Accès aux solutions, aux fournisseurs de services et aux partenaires des secteurs privé, public et des ONG qui peuvent les aider à développer et mettre en œuvre leurs stratégies de résilience

- L'adhésion à un réseau mondial de villes membres qui peuvent apprendre et s'entraider.

En outre, chaque ville du réseau bénéficie de l'aide d'un Chief Resilience Officer, nommé et finance par la fondation et responsable de la mise en place d'une politique de résilience. Ses objectifs sont de travailler avec les services municipaux des villes afin d'améliorer la communication entre les réseaux, réunir les acteurs concernés afin de les sensibiliser aux enjeux de la mise en place d'une ville résiliente, co-construire une stratégie globale de résilience et amorcer une réflexion pour développer une gouvernance développant les principes de résilience.

Le choix d'agir à l'échelle métropolitaine s'explique par la volonté de répondre à des enjeux précis, impact du changement climatique, inadéquation des transports, accessibilité à l'habitat, cohésion sociale, risques urbains n'en étant que des exemples. Soutenant à l'heure d'aujourd'hui 44 villes aussi diverses que Paris, San Francisco, El Paso, Glasgow, Amman, ou encore Tulsa, le consortium tente d'apporter des réponses adaptés et spécifiques aux problèmes que rencontrent ces villes suivant un modèle conceptuel de résilience. Définissant la résilience comme la capacité d'individus, de communautés, d'institutions, d'entreprises et de systèmes urbains à survivre, s'adapter et évoluer, peu importe les types de stress chroniques ou de chocs qu'ils peuvent rencontrer et défendant l'idée d'une approche holistique, le 100RC a construit un cadre définissant les caractéristiques de la résilience urbaine.

- Ateliers « les veilleurs de crue » (Gisclard, 2017)

Si les approches collaboratives peuvent s'effectuer à l'échelle des décideurs et gestionnaires locaux, la participation des habitants fait désormais partie du processus d'implémentation de la résilience sociale. L'appropriation du concept mais également des risques associés favorise le développement d'un savoir commun mais également d'un «bon » comportement en situation d'urgence. Béatrice Gisclard a développé (Gisclard, 2017) des ateliers collaboratifs pour mettre en scène un risque d'inondation du Vidourle à Sauve (Gard, France). Ces ateliers ont permis de développer un serious game (Pan et al., 2015; Patriarca et al., 2019; Taillandier and Adam, 2018) divisé en deux temps, «Sauve en 2030 » et «les $24 \mathrm{~h}$ de la crue ». Le serious game a eu pour objectif de mettre en situation une crue intense à horizon 2030 à Sauve et de mettre en perspective les actions individuelles et collectives à mettre en place au cours des premières $24 \mathrm{~h}$ de la catastrophe. Après avoir été divisés en équipes, les joueurs devaient piocher des cartes indiquant un contexte particulier (personne à mobilité réduite, jour férié, etc.), un moment (jour, nuit, matin, après-midi, etc.) et l'état des réseaux de communication (Fig.11). 


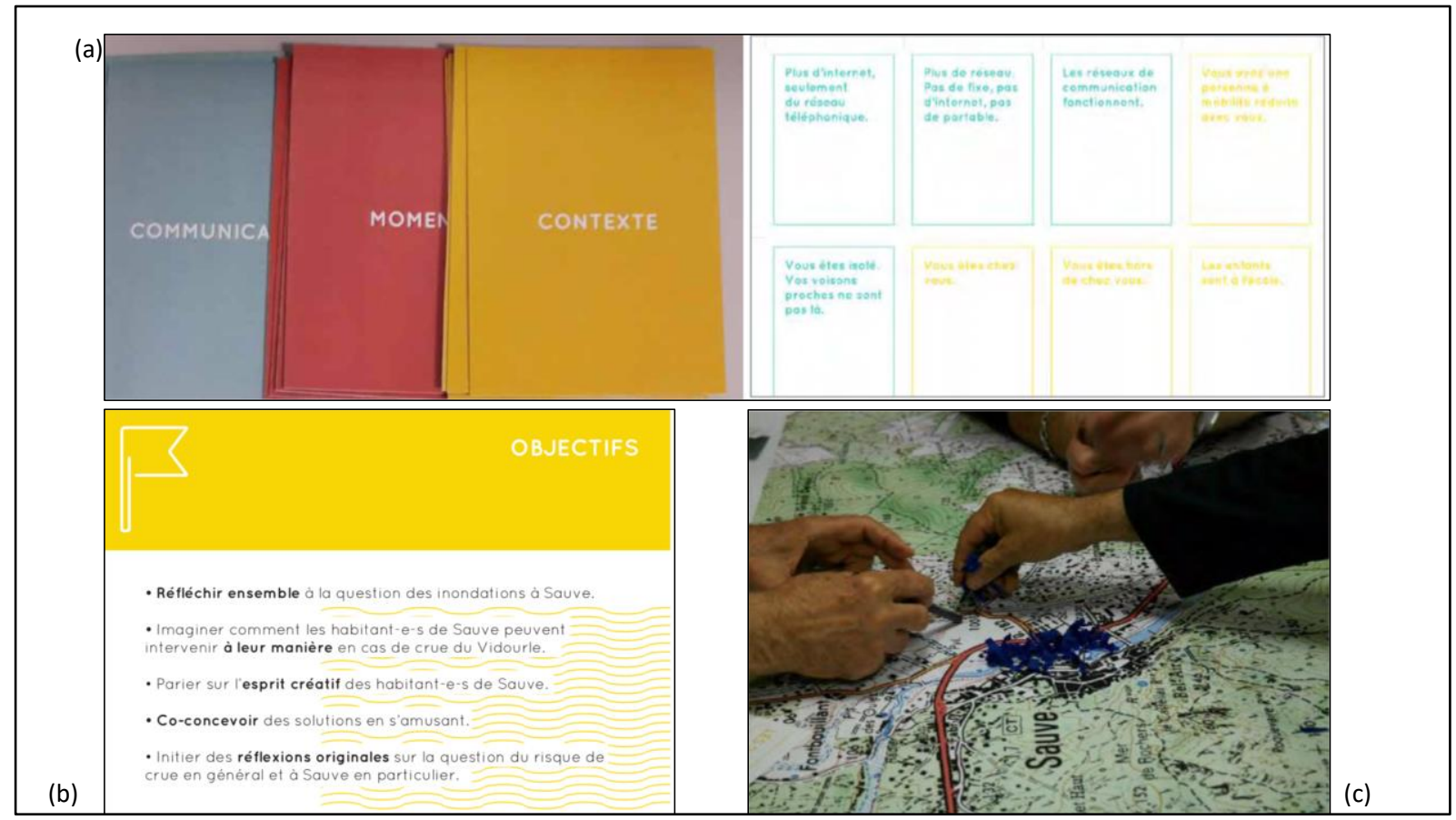

Figure 9. Ateliers de Sauve. (a) Les différentes cartes à jouer. (b) Les objectifs du jeu. (c) Les joueurs en action (Gisclard, 2017)

Les objectifs étaient multiples :

- Représenter la surface inondée en s'aidant de la mémoire collective et en s'appuyant sur les expériences d'inondations passées

- Matérialiser les déplacements envisagés

- Illustrer les comportements à adopter

Cet atelier a permis de contribuer à la conscience du risque mais également de la résilience en permettant une responsabilisation individuelle des comportements et actions à avoir en cas de crise. Afin de mettre en débat les problématiques liées à la gestion des risques, l'approche collective a été favorisée en permettant de développer un cadre commun d'expérience. De cette façon, chaque acteur, habitant, pouvait mettre en perspective son comportement et dépasser l'action purement individuelle.

Ce serious game a permis de responsabiliser les habitants et d'axer sur une résilience sociale collective à l'échelon des habitants. Premières victimes des catastrophes, les populations sont souvent peu intégrées au processus de gestion des risques et leur manque de connaissance conduit bien souvent à des comportements inappropriés et facteurs de sur-risques. Prendre en compte leurs expériences, leurs mémoires, leurs perceptions des risques et des enjeux est donc gage de résilience à moyen terme.

L'approche collaborative dans un processus d'aide à la décision permet de créer un lien entre les experts et les utilisateurs finaux. Intégrer les acteurs locaux au début de la conception d'un système spatial d'aide à la décision prend tout son sens lorsqu'il est question de sujets, thématiques et concepts controversés et complexes. Au-delà de la création d'une connaissance commune entre experts et acteurs, le processus collaboratif favorise l'acceptation et l'adoption d'outils, approches et méthodologies. Les outils existants dans la thématique des risques et de la résilience apportent mettent en lumière plusieurs atouts tels que le fait d'intégrer les acteurs permet de répondre à l'incompréhension du concept de résilience. En outre, l'approche collaborative pointe du doigt les limites préexistantes de la gestion cloisonnée des risques. Concevoir main dans la main une stratégie 
de résilience favorise donc l'acceptation et l'intégration du concept dans les pratiques locales de gestion des risques.

\section{Discussion}

Face à la difficile appropriation du concept de résilience, de nombreuses études ont tenté de fournir des méthodologies d'opérationnalisation (Tableau 1).

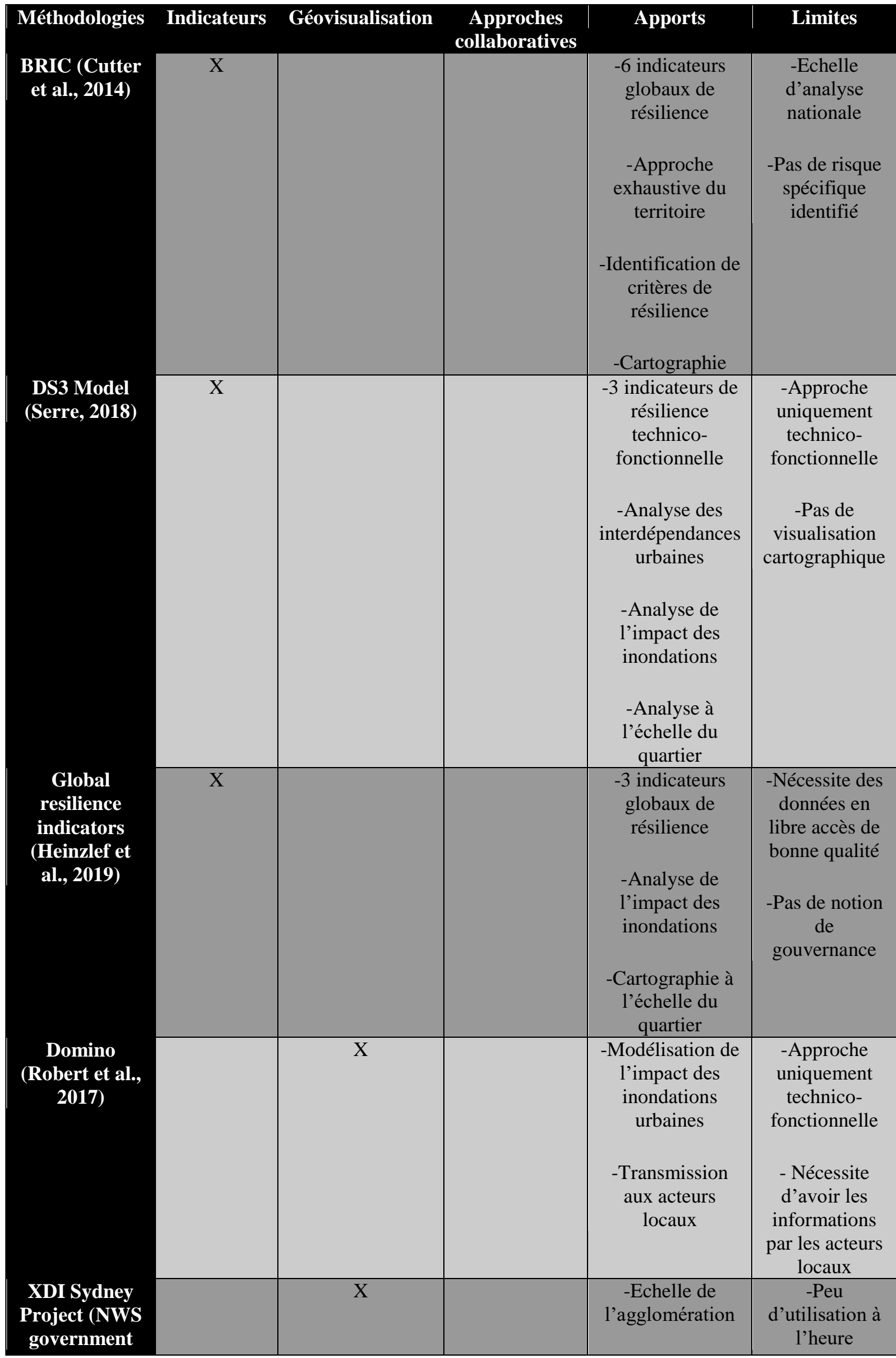




\begin{tabular}{|c|c|c|c|c|}
\hline $\begin{array}{c}\text { and } \\
\text { Australian } \\
\text { climate tech } \\
\text { company XDI } \\
\text { Cross } \\
\text { Dependency } \\
\text { Initiative, } \\
\text { 2018) }\end{array}$ & & & $\begin{array}{c}\text {-Cartographie } \\
\text { claire et } \\
\text { accessible } \\
\begin{array}{c}\text {-Prise en compte } \\
\text { des }\end{array} \\
\text { interdépendances } \\
\text { et des } \\
\text { infrastructures } \\
\text { critiques } \\
\text {-Volonté de } \\
\text { produire un } \\
\text { modèle global de } \\
\text { résilience } \\
\text {-Diversité } \\
\text { d'acteurs en jeu }\end{array}$ & $\begin{array}{l}\text { actuelle } \\
\text {-Centré sur } \\
\text { une seule ville } \\
\text {-Données non } \\
\text { accessibles }\end{array}$ \\
\hline $\begin{array}{l}\text { The coastline } \\
\text { resilience } \\
\text { (2007) }\end{array}$ & X & & $\begin{array}{c}\text {-Plusieurs } \\
\text { acteurs en jeu } \\
\\
\text {-Cartographie } \\
\text { claire et } \\
\text { accessible } \\
\\
\text {-Intégration de } \\
\text { plusieurs } \\
\text { composantes } \\
\text { (tells que la } \\
\text { vulnérabilité } \\
\text { sociale) }\end{array}$ & $\begin{array}{c}\text {-Peu de } \\
\text { transfert aux } \\
\text { habitants } \\
\text {-Données non } \\
\text { accessibles }\end{array}$ \\
\hline $\begin{array}{l}\text { Les } 100 \text { villes } \\
\text { résilientes } \\
\text { (The city } \\
\text { resilient } \\
\text { framework, } \\
\text { 2014) }\end{array}$ & & $\mathrm{X}$ & $\begin{array}{l}\text {-Approche } \\
\text { collaborative } \\
\text {-Dimension } \\
\text { globale de la } \\
\text { résilience } \\
\text {-Intégration } \\
\text { directe vers les } \\
\text { acteurs locaux }\end{array}$ & $\begin{array}{c}\text {-Pas de } \\
\text { données } \\
\text { accessibles } \\
\text {-Pas de } \\
\text { mesure de la } \\
\text { résilience }\end{array}$ \\
\hline $\begin{array}{c}\text { Ateliers les } \\
\text { Veilleurs de } \\
\text { crue } \\
\text { (Gisclard, } \\
\text { 2017) }\end{array}$ & & $\mathrm{X}$ & $\begin{array}{l}\text {-Action tournée } \\
\text { majoritairement } \\
\text { autour des } \\
\text { habitants } \\
\text {-Intégration de la } \\
\text { notion de jeu } \\
\text {-Implication des } \\
\text { habitants dans } \\
\text { les évenutelles } \\
\text { décision } \\
\text {-Appropriation } \\
\text { des enjeux }\end{array}$ & $\begin{array}{l}\text {-Ponctuel dans } \\
\text { le temps } \\
\text {-Longue mise } \\
\text { en place } \\
\text {-Pas de } \\
\text { mesure de } \\
\text { résilience } \\
\text {-Pas d'outil } \\
\text { récapitulatif }\end{array}$ \\
\hline
\end{tabular}


L'opérationnalisation du concept de résilience passe essentiellement par sa quantification à travers l'élaboration d'indicateurs de mesure. Ces indicateurs permettent d'établir des critères de résilience, des capacités à approfondir. L'objectif est d'établir une liste de caractéristiques à acquérir, augmenter et perpétuer.

L'élaboration d'indicateurs n'est que la première étape du processus. Afin de favoriser la compréhension et l'adoption de ces indicateurs, la conception de systèmes spatiaux d'aide à la décision s'appuyant sur les techniques de géovisualisation est nécessaire. La visualisation favorise le développement d'un savoir contextualisé et durable. L'abstraction du concept est moins perceptible. En outre les interfaces de manipulation permettent de s'approprier le concept et ses composants. Contextualiser et scénariser la résilience facilite la compréhension des enjeux, des tensions mais également des opportunités à développer.

Cette appropriation progressive ne peut s'établir sur le long terme que si une approche collaborative se développe. Favoriser l'investissement commun autour de problématiques telles que les risques et le changement climatique est une condition essentielle pour la construction d'une gestion des risques résiliente et sur le long terme. Confronter des savoirs et expériences d'horizons différents autour d'un enjeu tel que l'opérationnalisation de la résilience permet de diversifier et compléter les stratégies de résilience.

Malgré les avancées évidentes de ces approches, leur diversité et multitude pénalise une vision claire du concept et des méthodes d'opérationnalisation. Une boîte à outil rassemblant les approches existantes et permettant d'avoir une démarche consciente des enjeux et des outils pour y répondre. Ce prototype serait de promouvoir une approche inclusive qui permettrait de rassembler les différentes approches existantes autour du concept de résilience et de développer un cadre de réflexion et d'action entre les acteurs locaux et les experts scientifiques autour de la question de l'opérationnalisation du concept. Ce type d'outil pourrait être réalisé par la conception d'un observatoire de la résilience. Les observatoires sont des outils essentiels pour soutenir l'observation, la réflexion, la compréhension et l'analyse des phénomènes ou des territoires. Ces outils, qui sont à l'interface de la réalité et de la connaissance, sont essentiels dans le processus décisionnel, permettant l'acquisition de connaissances et de données tout en prenant la distance nécessaire pour avoir la vision la plus globale possible d'un phénomène. Leur utilité dans la mise en place d'un suivi des phénomènes, de l'évolution territoriale et de l'interaction, en font des outils essentiels pour appréhender les événements sur le long terme, ce qui est indispensable à la mise en place de stratégies de résilience. Une équipe basée à l'unité mixte de recherche sur les écosystèmes insulaires océaniques (UMR EIO) de l'Université de Polynésie française (Heinzlef et al., 2020a) a lancé un prototype d'observatoire sur les îles de Tahiti et Moorea pour analyser, mesurer et opérationnaliser la résilience. Les objectifs sont multiples et portent notamment sur l'amélioration de la connaissance des risques territoriaux, l'acquisition, le stockage et la valorisation des données relatives aux risques et à la résilience et enfin l'intégration des acteurs dans le processus de réflexion et de mise en œuvre des stratégies de résilience. Ce prototype peut servir de base de réflexion et de suggestions pour la poursuite de la mise en œuvre progressive du concept de résilience dans les stratégies de gestion des risques.

\section{Conclusion}

Cet article développe les enjeux des risques en milieux urbains et de l'intégration progressive du concept de résilience dans leur gestion. Concept aux multiples origines, le concept de résilience est une des réponses à l'augmentation des risques et des incertitudes, favorisant des stratégies évolutives, adaptives et systémiques. Pourtant, la résilience demeure une notion abstraite et peu appropriée par les acteurs, décisionnaires et manageurs locaux. 
De multiples études ont permis de développer des approches d'opérationnalisation de la résilience. Ces stratégies d'opérationnalisation peuvent promouvoir la conception d'indicateurs pour définir et mesurer la résilience, développer des systèmes d'aide à la décision spatiale pour visualiser la résilience territoriale ou promouvoir la mise en œuvre d'approches collaboratives pour impliquer les acteurs locaux dans l'intégration du concept dans les stratégies locales de gestion des risques. Bien que ces méthodologies offrent en elles-mêmes des possibilités de réflexion ou même des initiatives en matière de stratégies de résilience, leur contribution reste modeste et visible dans un laps de temps très court.

Réfléchir à un nouveau type d'outil pour aborder la résilience à long terme et à une approche inclusive du concept et des méthodologies associées permettrait de répondre à ces limitations actuelles. Cet outil, qui prendrait la forme d'un observatoire de la résilience, permettrait de développer une boîte à outils, rassemblant les avancées conceptuelles et tangibles liées à l'opérationnalisation de la résilience.

\section{Remerciements}

Cette publication a reçu le soutien financier du CNRS à travers les programmes interdisciplinaires du MITI et de l'IRD.

\section{Références}

Adger, W. N., Brooks N., Bentham G., Agnew M., et Eriksen S. New indicators of vulnerability and adaptive capacity. $\mathrm{V}$ ol. 122. $\mathrm{T}$ yndall Centre for Climate Change Research Norwich, 2004. http://www.tyndall.ac.uk/sites/default/files/Adger\%20W.\%20N\%20.,\%20Brooks,\%20N.\%20,\%20Kelly,\%20M.,\% 20Bentham,\%20S.\%20and\%20Eriksen,\%20S.\%20(2004)\%20New\%20indicators\%20of\%20 vulnerability\%20and\%20adaptive\%20capacity\%20(tr7).pdf.

Albouy, F.-X., 2002. Le temps des catastrophes, Interface/econo. Descartes, Paris.

Bahadur, A., Wilkinson E., et Tanner T. « Measuring Resilience: An Analytical Review (Draft under Review) ». Unpublished, 2015. https://doi.org/10.13140/RG.2.1.1300.1444.

Bahadur, A.V., Ibrahim, M., Tanner, T., 2010. The resilience renaissance? Unpacking of resilience for tackling climate change and disasters 45 .

Balsells, M., 2015. Contribution de la conception d'un quartier à la résilience urbaine face au risque d'inondation à l'échelle du quartier. Thèse de doctorat. Université de Mons-Faculté d'Architecture et d'Urbanisme, Mons.

Barroca, B. « Résilience Urbaine: Une venelle entre impasse et avenu(e). Habilitation à Diriger les Recherches. Volume 2 ». Université Paris-Est, 2018.

Bercerra, S., et Peltier A., éd. Risques et environnement, recherches interdisciplinaires sur la vulnérabilité des sociétés. Sociologies et environnement. Paris: L'Harmattan, 2009.

Boin, A., McConnell, A., 2007. Preparing for critical infrastructure breakdowns: the limits of crisis management and the need for resilience. J. Contingencies Crisis Manag. 15, 50-59.

Bonnefous, C. Indicateurs de performance. Relié. Ic2 productique. Paris: Hermès Science Publ., 2001.

Booth, J.W., et Neill J.T. « Coping Strategies and the Development of Psychological Resilience ». Journal of Outdoor and Environmental Education 20, $\mathrm{n}^{\mathrm{O}} 1$ (2017): 47-54. https://doi.org/10.1007/BF03401002.

Bretagnolle, A., Daudé E., et Pumain D. « From theory to modelling: urban systems as complex systems ». Cybergeo, 2006. https://doi.org/10.4000/cybergeo.2420.

Burton, I., Kates R.W., et White G.F. The environment as hazard. 2nd ed. New York: Guilford Press, 1993.

Campanella, T.J., 2006. Urban Resilience and the Recovery of New Orleans. J. Am. Plann. Assoc. 72, 141-146. https://doi.org/10.1080/01944360608976734 
Cardona, O.D., Van Aalst M.K., Birkmann J., Fordham M., McGregor G., Perez R., Pulwarty R.S., et al. «Determinants of Risk: Exposure and Vulnerability ». In Managing the Risks of Extreme Events and Disasters to Advance Climate Change Adaptation, édité par Christopher B. Field, Vicente Barros, Thomas F. Stocker, et Qin Dahe, 65-108. Cambridge: Cambridge University Press, 2012. https://doi.org/10.1017/CBO9781139177245.005.

Catastrophes Naturelles-Observatoire permanent des catastrophes naturelles et des risques naturels. «Bilan statistique des catastrophes naturelles survenues dans le Monde entre 2002-2015», 2016. https://www.catnat.net/donneesstats/dernieres-actualites/21507-bilan-statistique-des-catastrophes- naturellessurvenues-dans-le-monde-entre-2001-2015-2.

Coastline Resilience Project, 2007. https://maps.coastalresilience.org

Çöltekin, A., Bleisch, S., Andrienko, G., Dykes, J., 2017. Persistent challenges in geovisualization - a community perspective. Int. J. Cartogr. 3, 115-139. https://doi.org/10.1080/23729333.2017.1302910

Constas, M.A., Frankenberger T.R., et Hoddinott J. « Resilience measurement principles toward an agenda for measurement design ». FAO, WFP, 2010. https://www.fsnnetwork.org/resilience-measurement- principles-towardagenda-measurement-design.

Cutter, S.L., Ash K.D., et Emrich C.T. « The Geographies of Community Disaster Resilience ». Global Environmental Change 29 (2014): 65-77. https://doi.org/10.1016/j.gloenvcha.2014.08.005.

Cutter, S.L., Burton C.G., et Emrich C.T. « Disaster Resilience Indicators for Benchmarking Baseline Conditions». Journal of Homeland Security and Emergency Management 7, $\mathrm{n}^{\mathrm{O}} 1$ (2010). https://doi.org/10.2202/15477355.1732.

Cutter, S. L., et Finch C. «Temporal and Spatial Changes in Social Vulnerability to Natural Hazards ». Proceedings of the National Academy of Sciences 105, no 7 (2008): 2301-6. https://doi.org/10.1073/pnas.0710375105

Cutter, S.L., Barnes L., Berry M., Burton C., Evans E., Tate E., et Webb J. « A Place-Based Model for Understanding Community Resilience to Natural Disasters ». Global Environmental Change 18, n 4 (2008): 598-606. https://doi.org/10.1016/j.gloenvcha.2008.07.013.

Cutter, S.L., Barnes, L., Berry, M., Burton, C., Evans, E., Tate, E., Webb, J., 2008. Community and Regional Resilience: Perspectives from Hazards, Disasters, and Emergency Management (Carri Research Report No. 1). Community and Regional Initiative.

Cutter, S.L., Boruff B.J., et Shirley W.L. « Social Vulnerability to Environmental Hazards* ». Social Science Quarterly 84, no 2 (2003): 242-61. https://doi.org/10.1111/1540-6237.8402002.

Cyrulnik, B., 2004. La Résilience ou comment renaître de sa souffrance?, Penser le monde de l'enfant. Fabert.

Dauphiné, A, et Provitolo D. Risques et catastrophes observer, spatialiser, comprendre, gérer. Paris: A. Colin, 2013.

Dauphiné, A., Provitolo, D., 2007. La résilience : un concept pour la gestion des risques. Ann. Géographie 654, 115. https://doi.org/10.3917/ag.654.0115

Dauphiné, A., Provitolo, D., 2004. Risques et catastrophes - Observer, spatialiser, comprendre, gérer [WWW Document]. Armand Colin. URL http://www.armand-colin.com/risques-et-catastrophes-observer-spatialisercomprendre-gerer-9782200278427 (accessed 1.20.17).

Desthieux, G. « Approche systémique et participative du diagnostic urbain. Processus de représentation cognitive du système urbain en vue de l'élaboration d'indicateurs géographiques ». Ecole Polytechnique fédérale de Lausanne, 2005.

Djament-Tran, G., et Reghezza-Zitt M.. Résiliences urbaines: les villes face aux catastrophes. Le Manuscrit. Recherche et Université. Paris, 2012.

Djament-Tran, G., Le Blanc, A., Lhomme, S., Rufat, S., Reghezza-Zitt, M., 2011. Ce que la résilience n'est pas, ce qu'on veut lui faire dire.

Duvat-Magnan, V., et Magnan A. Des catastrophes ... naturelles? Paris: Pommier, 2014.

Emrich, C.T., Tobin, G.A., 2018. Resilience: An Introduction, in: Vulnerability and Resilience to Natural Hazards. Cambridge University Press, pp. 124-144.

Flanagan, B.E., Gregory E.W., Hallisey E.J., Heitgerd J.L., et Lewis B. « A Social Vulnerability Index for Disaster Management». Journal of Homeland Security and Emergency Management $8, \mathrm{n}^{\mathrm{O}} \quad 1 \quad$ (2011). https://doi.org/10.2202/1547-7355.1792. 
Galland, J-P. «Critique de la notion d'infrastructure critique ». Flux, $\mathrm{n}^{\mathrm{O}} 81$ (2010): 6-18.

Gardner, G. « The City: A System of Systems ». In State of the World, par Worldwatch Institute, 27-44. Washington, DC: Island Press/Center for Resource Economics, 2016. https://doi.org/10.5822/978-1- 61091-756-8_3.

Adger, W.N., Brooks, N., 2003. Does global environmental change cause vulnerability to disaster?, in: Natural Disaster and Development in a Globalizing World. London, p. 24.

Albouy, F.-X., 2002. Le temps des catastrophes, Interface/econo. Descartes, Paris.

Alexander, D.E., 2013. Resilience and disaster risk reduction: an etymological journey. Nat. Hazards Earth Syst. Sci. Discuss. 1, 1257-1284. https://doi.org/10.5194/nhessd-1-1257-2013

Barroca, B., Bernardara, P., Mouchel, J.M., Hubert, G., 2006. Indicators for identification of urban flooding vulnerability. Nat. Hazards Earth Syst. Sci. 6, 553-561. https://doi.org/10.5194/nhess-6-553-2006

Boin, A., McConnell, A., 2007. Preparing for critical infrastructure breakdowns: the limits of crisis management and the need for resilience. J. Contingencies Crisis Manag. 15, 50-59.

Dauphiné, A., Provitolo, D., 2013. Risques et catastrophes observer, spatialiser, comprendre, gérer. A. Colin, Paris.

Dobson, M., Smith, P., Evans, H., Chatterton, J., 2016. The Holistic Integrity Test (HIT) - quantified resilience analysis. E3S Web Conf. 7, 08012. https://doi.org/10.1051/e3sconf/20160708012

Gersonius, B., 2012. The resilience approach to climate adaptation applied for flood risk. CRC, Leiden.

Gersonius, B., Ashley, R., Pathirana, A., Zevenbergen, C., 2013. Climate change uncertainty: building flexibility into water and flood risk infrastructure. Clim. Change 116, 411-423. https://doi.org/10.1007/s10584-012-0494-5

Gisclard, B., 2017. L'innovation sociale territorialisée: un levier de réappropriation du risque inondation par les habitants. Université d'Avignon et des pays de Vaucluse.

Gonzva, M., Balsells, M., Barroca, B., 2015. Mobilisation de l'analyse fonctionnelle pour l'étude de la résilience des quartiers et des systèmes de transport guidé face au risque inondation. Tech. Sci. Méthodes 64-75. https://doi.org/10.1051/tsm/201507064

Gonzva, M., Barroca, B., Gautier, P.-É., Diab, Y., 2017. Modeling disruptions causing domino effects in urban guided transport systems faced by flood hazards. Nat. Hazards 86, 183-201. https://doi.org/10.1007/s11069-016-2680-7

Helbing, D., 2013. Globally networked risks and how to respond. Nature 497, 51-59. https://doi.org/10.1038/nature12047

Lhomme, S., Serre, D., Diab, Y., Laganier, R., 2013. Analyzing resilience of urban networks: a preliminary step towards more flood resilient cities. Nat. Hazards Earth Syst. Sci. 13, 221-230. https://doi.org/10.5194/nhess-13221-2013

Mancebo, F., 2006. Katrina et la Nouvelle-Orléans : entre risque «naturel» et aménagement par l'absurde. Cybergeo. https://doi.org/10.4000/cybergeo.90

Meerow, S., Newell, J.P., Stults, M., 2016. Defining urban resilience: A review. Landsc. Urban Plan. 147, 38-49. https://doi.org/10.1016/j.landurbplan.2015.11.011

Merad, M., Chojnacki, E., Rouïl, L., 2016. Prise en compte des incertitudes dans la cartographie des risques Quelques recommandations dans le cas de la prévention des pollutions atmosphériques, in: Congrès Lambda Mu 20 de Maîtrise Des Risques et de Sûreté de Fonctionnement. Presented at the Congrès Lambda Mu 20 de Maîtrise des Risques et de Sûreté de Fonctionnement, 11-13 Octobre 2016, Saint Malo, France, IMdR. https://doi.org/10.4267/2042/61762

Nones, M., Pescaroli, G., 2016. Implications of cascading effects for the EU Floods Directive. Int. J. River Basin Manag. 14, 195-204. https://doi.org/10.1080/15715124.2016.1149074

Pan, J., Su, X., Zhou, Z., 2015. An Alternate Reality Game for Facility Resilience (ARGFR). Procedia Eng. 118, $296-$ 303. https://doi.org/10.1016/j.proeng.2015.08.430

Patriarca, R., Falegnami, A., De Nicola, A., Villani, M.L., Paltrinieri, N., 2019. Serious games for industrial safety: An approach for developing resilience early warning indicators. Saf. Sci. 118, 316-331. https://doi.org/10.1016/j.ssci.2019.05.031

Pescaroli, G., Alexander, D., 2015. A definition of cascading disasters and cascading effects: Going beyond the "toppling dominos" metaphor. Planet@Risk 3, 58-67. 
Pescaroli, G., Kelman, I., 2017. How Critical Infrastructure Orients International Relief in Cascading Disasters. J. Contingencies Crisis Manag. 25, 56-67. https://doi.org/10.1111/1468-5973.12118

Reghezza-Zitt, M., 2017. Penser la vulnérabilité dans un contexte de globalisation des risques grâce aux échelles spatiales et temporelles. Espace Popul. Sociétés. https://doi.org/10.4000/eps.6641

Serre, D., Heinzlef, C., 2018. Assessing and mapping urban resilience to floods with respect to cascading effects through critical infrastructure networks. Int. J. Disaster Risk Reduct. https://doi.org/10.1016/j.ijdrr.2018.02.018

Taillandier, F., Adam, C., 2018. Games Ready to Use: A Serious Game for Teaching Natural Risk Management. Simul. Gaming 49, 441-470. https://doi.org/10.1177/1046878118770217

Zevenbergen, C., Cashman, A., Evelpidou, N., Pasche, E., Garvin, S., Ashley, R., 2010. Urban Flood Management. CRC Press.

Zevenbergen, C., Cashman A., Evelpidou NE. Pasche E., Garvin S., et Ashley R. Urban Flood Management. CRC Press, 2010. 